\title{
Effect of Isotherm Shape on Mass Transfer in an Adsorbent Particle; An Isothermal Adsorption Process
}

\author{
Zeynep Elvan Yildirim ${ }^{*}, 1$, Gamze Gediz Ilis ${ }^{2}$, Moghtada Mobedi ${ }^{2}$ and Semra Ülkü ${ }^{3}$ \\ ${ }^{I}$ Energy Engineering Program, Izmir Institute of Technology, Urla 35410 Izmir, Turkey \\ ${ }^{2}$ Mechanical Engineering Department, Izmir Institute of Technology, Urla 35410 Izmir, Turkey \\ ${ }^{3}$ Chemical Engineering Department, Izmir Institute of Technology, Urla 35410 Izmir, Turkey
}

\begin{abstract}
The mass diffusion equation and corresponding initial and boundary conditions were non-dimensionalized for a spherical adsorbent particle in an infinite adsorptive medium to reduce number of independent governing parameters into two as mass transfer Biot number and dimensionless isotherm. The changes in local and average adsorbate concentration throughout the adsorption process were calculated and plotted for different values of mass transfer Biot numbers (ranging from 0.5 to 750 ) and for five different isotherm shapes. The obtained results indicated that the isotherm shape influences dimensionless period of adsorption, if the external mass transfer resistance is significant in the process. For those cases, the total dimensionless adsorption period for a convex shape isotherm is shorter than a concave one.
\end{abstract}

Keywords: Isotherm shape, mass transfer, physical adsorption, solid diffusion.

\section{INTRODUCTION}

Adsorption is simply the transfer of a fluid to a solid surface where it is held by attraction forces between the solid and the molecules of the fluid. Related to the nature of forces involved, the phenomena may be called as chemical or physical adsorption. Although in the case of chemical adsorption, chemical bond is formed between the sorbate and the adsorbent resulting from the transfer of electrons; in physical adsorption there is no chemical bond formation and van der Waals forces are responsible for the adsorption. If either the solid or the fluid is polar in nature, contributions of the electrostatic forces will also be arising due to polarization, dipole, and quadrupole interactions.

Adsorption phenomena have broad range of application in the nature as well as in the industry. It is widely faced in the catalytic reactions and in the separation/purification processes. The recovery of the chemical compounds, water purification, separation and purification of air, drying, medical treatments, and recently thermally driven energy systems are among its applications which we come across in the daily life. For a specific application, the selection of a proper adsorbent is the most crucial step. Factors such as the selectivity and capacity of the adsorbent, affinity of the adsorbent /adsorptive pair for each other, the rate and the reversibility of the process are among the prime concerns of the decision makers for a specific industrial application of adsorption. While studies related to the improvement of the well known classical adsorbents (such as zeolite, silica gel, active carbon) are going on, there are efforts for finding new pairs such as carbon nanotubes, nanohorns, activated carbon

*Address correspondence to this author at the Energy Engineering Program, Izmir Institute of Technology, Urla 35410 Izmir, Turkey; Tel: +902327506744; Fax: +902327506701; E-mail: zeynepyildirim@iyte.edu.tr fibers [1-3]. The diffusion rate of an adsorbate in an adsorbent particle can be determined based on Fick's law. For an isothermal adsorption process, the differential mass balance equation, adsorption equilibrium relationship together with the initial and the boundary conditions are employed to determine the change of adsorbate concentration in the particle. The effective mass diffusivity may not be constant during adsorption and it may vary with adsorbate concentration.

In the literature, there are not many detailed studies on the effect of isotherm shape on adsorption period or adsorption rate. Erkey et al. [4] investigated the influence of isotherm shape on adsorption and desorption profiles of volatile organic contaminants from oven-dried soil in a packed bed column. They showed the strong influence of isotherm on both adsorption and desorption breakthrough profiles. San and Lin [5] performed a theoretical study to compare three adsorption pairs having different isotherms (activated carbon-methanol, silica gel-water, and 13X molecular sieves - water) for using as working substances in a multi-bed adsorption heat pump. They found that among the three considered adsorption pairs, the activated carbonmethanol pair possesses the highest vapor pressure in the adsorber. Due to the higher vapor pressure larger overall heat transfer coefficient between the adsorptive and adsorbent were obtained, and consequently the cycle time was decreased. Nobrega and Brum [6] performed a theoretical study on the influence of isotherm shape over desiccant cooling cycle performance. Three different desiccant materials were represented by a generalized adsorption isotherm, which was characterized by a single parameter. The analysis revealed that silica gel exhibits better performance than Zeolite 4A particularly for low regeneration temperatures. Heat and mass transfer in a single adsorbent particle with negligible and/or considerable external heat and mass transfer resistances are discussed by Karger and Ruthven [7, 8]. Generally, 
the dimensional form of heat and mass transfer equations for a single particle are considered in these text books. Suzuki [9] studied the dimensionless form of heat and mass transfer equations for a single spherical particle. However; detailed discussions on the effects of isotherm shape and external mass transfer resistance on the change of adsorbate concentration in the particle and adsorption period were not performed.

The aim of present study is to perform a theoretical work to investigate the effect of isotherm shape on the total dimensionless adsorption period of an isothermal physical gas adsorption process. The study is performed for the adsorption cases in which the adsorbed gas (i.e., adsorbate) is transferred uniformly from the surface to the center of adsorbent particle by surface (or solid) diffusion mechanism. In order to reduce number of governing parameters, mass diffusion equation for a spherical adsorbent particle and the related initial and boundary conditions are non-dimensionlized. The number of governing parameters is reduced to two parameters as mass transfer Biot number and dimensionless isotherm equation which provides a relation between dimensionless adsorbate concentration and dimensionless adsorptive density. The mass diffusion equation is numerically solved for different values of mass transfer Biot number and isotherm shapes. Based on the obtained results, variation in local adsorbate concentration profiles and the changes in average dimensionless adsorbate concentration over dimensionless time are plotted, and the effects of isotherm shape on the total dimensionless adsorption period are discussed in details.

\section{ADSORPTION EQUILIBRIA}

The maximum amount of vapor taken up by an adsorbent at a specified temperature and pressure is determined by adsorption equilibria. Adsorption equilibrium relationship at constant temperature (i.e., adsorption isotherm) provides a

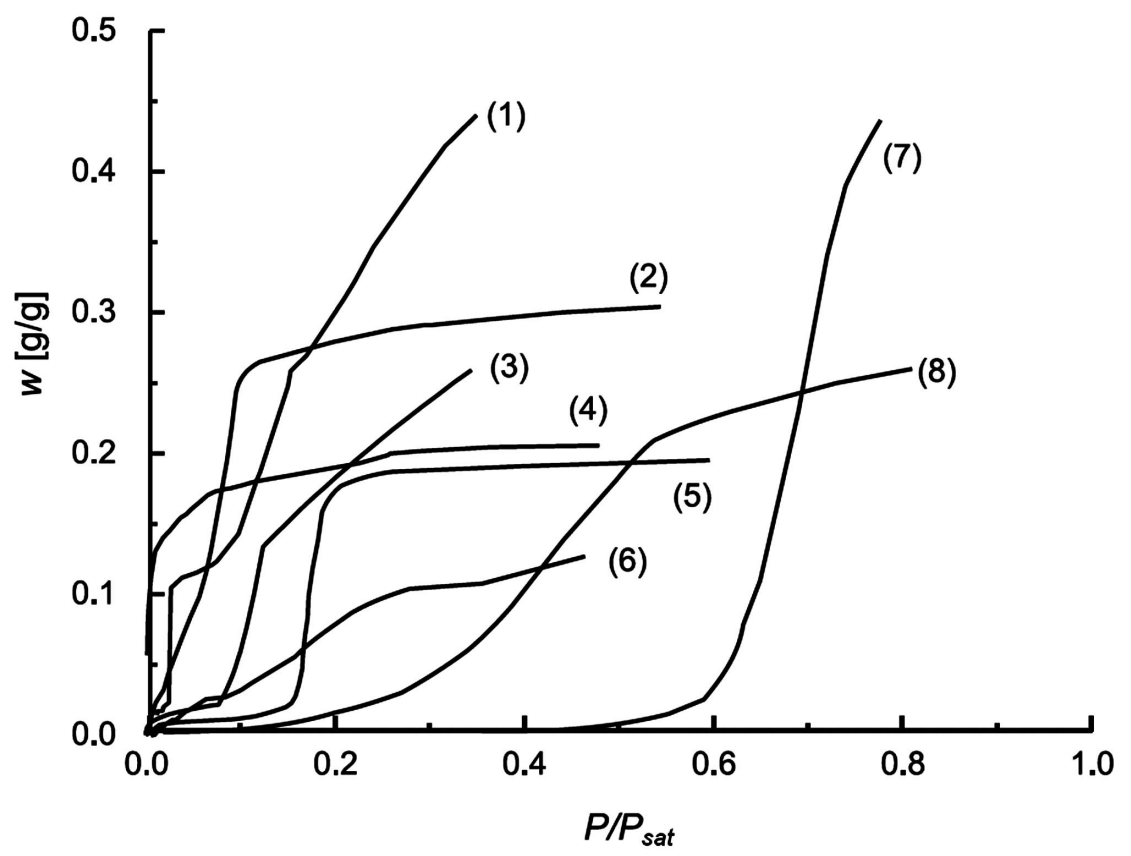

relationship between equilibrium concentrations of adsorbate and adsorptive. The equilibrium condition between adsorbent and adsorbate can be estimated by various theoretical and experimental methods. Fig. (1) shows the isotherm shapes of different adsorbents-water vapor pairs. As seen, the shapes of isotherms are quite different from each others. Isotherms of FAMZ02-water and Zeolite 13X-water have convex shape, isotherm of Carbon Aerogel a-CA-54 - water has concave shape while isotherms of Silica Fuji Silys RDwater has a linear configuration. Considering the possible effect of adsorption isotherm shape on the adsorption period, the isotherm shape will be guiding in the selection of a proper pair.

Different shapes of isotherms exist based on the nature and affinity of adsorbent-adsorbate pairs. Although various shapes of adsorption isotherms were reported in the literature; six basic standard adsorption isotherms, defined by International Union of Pure and Applied Chemistry (i.e., IUPAC), are shown in Fig. (2). Adsorbents with pore sizes approximately equal to the molecule size of adsorbate comply with type I isotherm. It is the characteristic isotherm shape for molecular sieves zeolites. Types II and IV define the adsorption which occurs in the adsorbents with dominant macropores. Types III and V isotherms do not exhibit knee shape behavior on their curves which is an indicative of weak adsorbent-adsorbate interactions [10]. The stepped isotherm, appropriately designated Type VI and relatively rare, is of particular theoretical interest [11]. In gas adsorption, although the adsorptive concentration is usually expressed in terms of pressure, in this study for simplicity, adsorptive concentration is given in terms of adsorptive density. The isotherm based on density variation can be easily obtained from adsorptive pressure by using ideal gas law under low pressure isothermal process conditions.

Adsorption equilibrium relationship determines the adsorbate concentration at the outer surface of an adsorbent particle, and it is generally represented by an isotherm (i.e. 
equilibrium concentrations under constant temperature). For a gas phase adsorption at the specified temperature, the relationship between adsorbate concentration in the adsorbent particle and adsorptive pressure (i.e., gas or vapor pressure) is generally preferred to describe equilibrium condition. There exist numbers of adsorption equilibrium models; Henry Law, Langmuir, Dubinin-Astakhov, and Freundlich are the ones which are frequently used. Henry Law with linear relationship which is valid for low concentrations is preferred due to its simplicity. An adsorption isotherm for a specific case can be obtained experimentally. The obtained experimental data can be used to find the best fit model for the considered adsorption process.

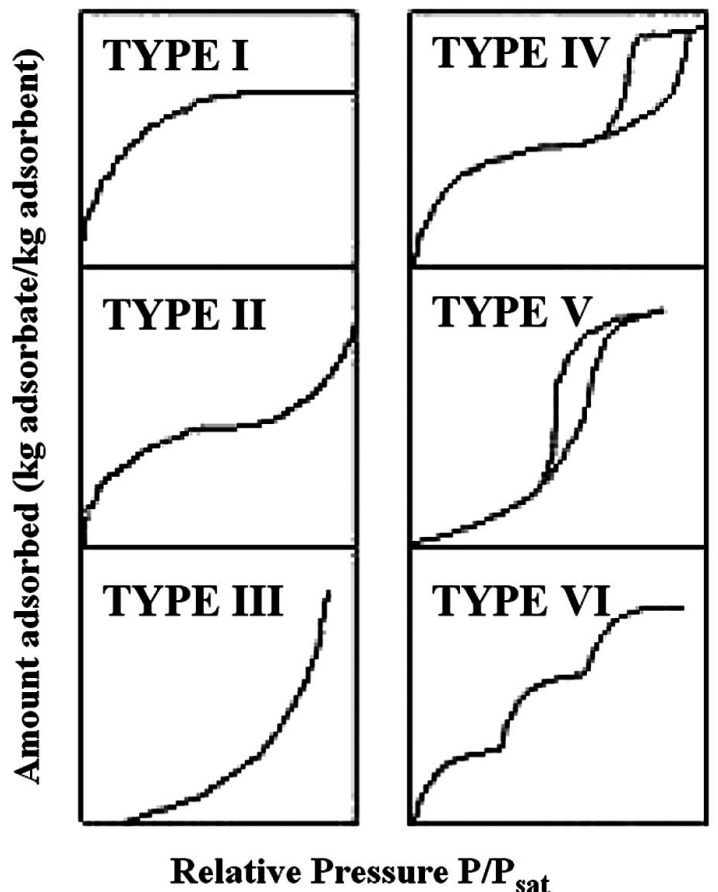

Fig. (2). Types of adsorption isotherms based on IUPAC classification.

\section{THE STUDIED ADSORBENT PARTICLE}

Fig. (3) shows a schematic view of the analyzed adsorbent particle. Fig. (3a) shows the dimensional quantities for the studied adsorbent particle while Fig. (3b) illustrates the dimensionless quantities. It is assumed that the adsorbent particle is located in an infinite adsorptive media. It has a spherical shape with a radius of $r_{p}$. The pressure, density, and temperature of the adsorptive are $P_{\infty}, \rho_{\infty}$ and $T_{\infty}$ which are constant during the adsorption. The process is isothermal and the surface of adsorbent particle is at equilibrium. The external mass transfer resistance at the outer region of adsorbent particle causes a density gradient around the particle and consequently the adsorptive density at the outer surface of particle becomes different than the density of the surrounding vapor. In this study, it is assumed that Fick's law is valid for adsorbate diffusion from the surface to the center of the adsorbent particle and the drive force for adsorbate transfer through the adsorbent particle is only the adsorbate concentration gradient in radial direction. The adsorbate concentration is defined based on the weight of adsorbate in the dry adsorbent particle (i.e., $W$ ). Moreover, the mass diffusivity is assumed constant, and it can be represented by effective mass diffusivity (i.e., $D_{\text {eff }}$ ). The effective mass diffusivity (or apparent mass diffusivity) involves all mass transfer mechanisms such as such as surface, pore diffussion etc in the adsorbent particle.
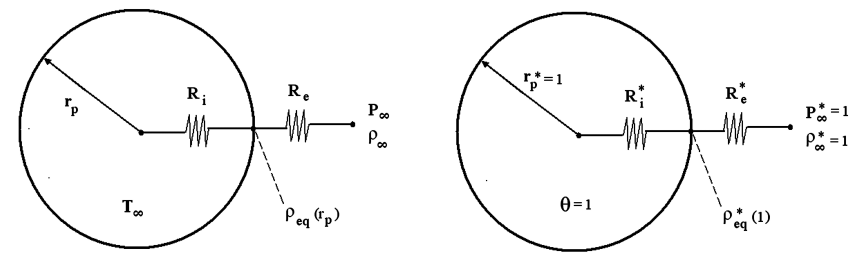

Fig. (3). Schematic view of analyzed adsorbent particle (a) dimensional quantities (b) dimensionless quantities.

The dimensionless form of the isotherm equation yields a relation between dimensionless adsorptive density $\left(\rho_{v}^{*}\right)$ and dimensionless adsorbate concentration $\left(W^{*}\right)$;

$W^{*}=\left(\rho_{v}^{*}\right)^{n}$

where;

$W^{*}=\frac{W}{W_{\infty}}, \rho_{v}^{*}=\frac{\rho_{v}}{\rho_{\infty}}$

Here, $\rho_{\infty}$ is the adsorptive density and $W_{\infty}$ represents the equilibrium adsorbate concentration at $\rho_{\infty}$ and $T_{\infty}$. As it was shown in Fig. (1), many shapes of isotherms exit. The shape of them can be convex, concave, linear, etc. Eq. (1) is used to generate different dimensionless isotherm shapes since the aim of present study is to show the effect of isotherm shape on dimensionless adsorption period. It should be mentioned that the present study is not performed for a specific adsorbent-adsorbate pair. The coefficient of $n$ is a constant related to the shape of the isotherm. The isotherm with $n=0.05$ has a convex shape while a concave shape is seen for the isotherm with $n=20$. The present study was performed for different $n$ values $(0.05,0.5,1$, and 20). Fig. (4) depicts the isotherm shapes studied in the present work.

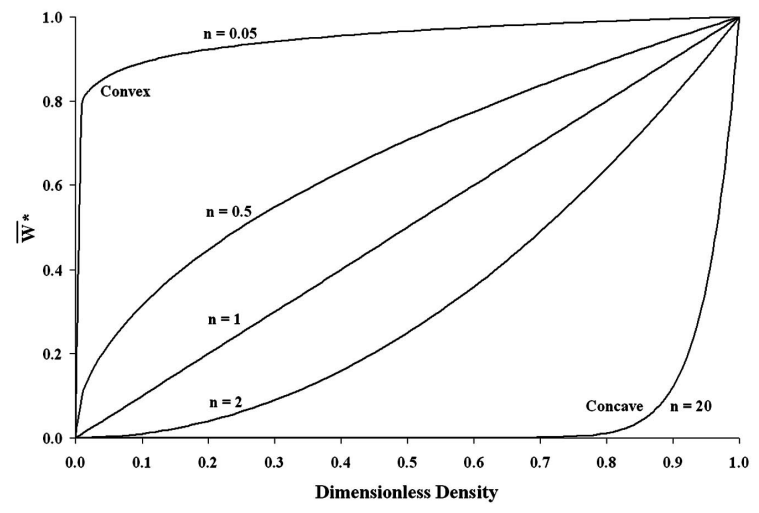

Fig. (4). Different shapes of studied isotherms.

The dimensionless form of isotherms is useful diagrams since it can unify the isotherms of various adsorbent-adsor- 
bate pairs. Fig. (5) shows the dimensional and dimensionless isotherms of silica gel - water and active carbon - ammonia pairs for 40 and $60{ }^{\circ} \mathrm{C}$, respectively. The isotherm equations were plotted based on the relations given by Daou et al. [12] and Tamainot-Telto et al. [13] respectively. In Fig. (5b), the adsorbate concentration is non-dimensionlized by using Eq. (2). The equilibrium adsorbate concentration for silica gel water at $40{ }^{\circ} \mathrm{C}$ and Active carbon -ammonia at $60{ }^{\circ} \mathrm{C}$ are $0.448(\mathrm{~kg} / \mathrm{kg})$ and $0.81(\mathrm{~kg} / \mathrm{kg})$, respectively. As seen, although dimensional isotherms of two pairs are different from each other, the dimensionless isotherms are identical. Hence, the results of a dimensionless study based on dimensionless isotherm of Fig. (5b) are valid for both adsorbent-adsorbate pairs, if the adsorbate transport can be renresented hv the same mass transfer mechanism

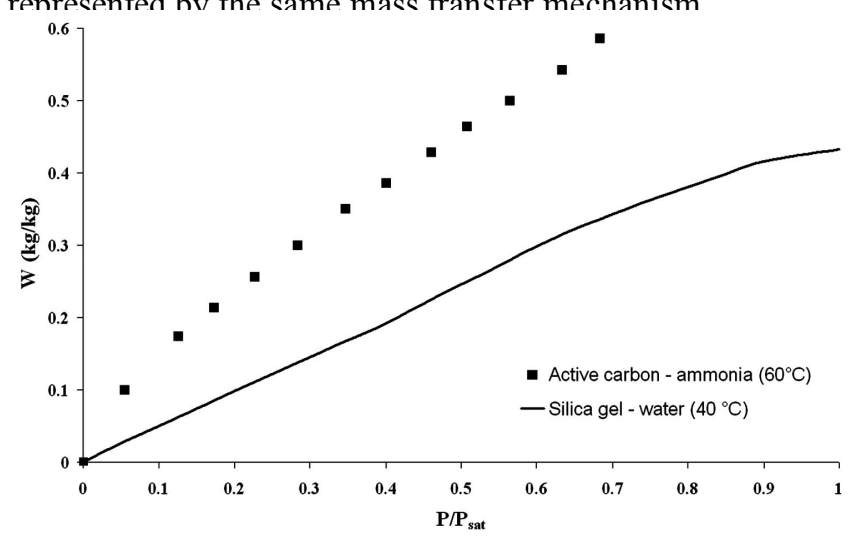

(a)

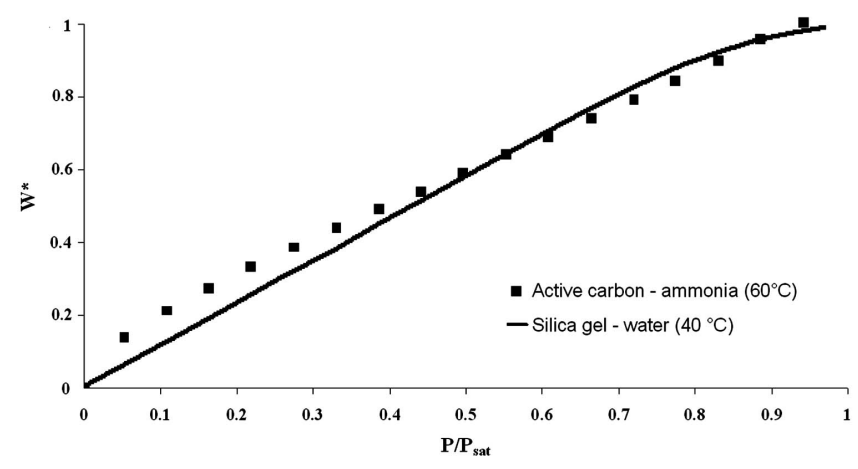

(b)

Fig. (5). Isotherm graphs of silica gel - water pair at $40{ }^{\circ} \mathrm{C}$ and active carbon - methanol pair at $60{ }^{\circ} \mathrm{C}$. a) dimensional b) dimensionless.

\section{MASS DIFFUSION EQUATION}

Considering the assumptions explained in previous section, for a surface diffusion (or solid diffusion) of adsorbate in an adsorbent particle, the following partial differential equation can be written to obtain the distribution of the adsorbate concentration in the adsorbent particle during the adsorption process.

$\frac{\partial W}{\partial t}=\frac{1}{r^{2}} \frac{\partial}{\partial r}\left(r^{2} D_{e f f} \frac{\partial W}{\partial r}\right)$

The initial and boundary conditions of Eq. (3) can be written as;
At $r=0$

$$
\frac{\partial W}{\partial r}=0
$$

At $r=r_{p}$

$$
-D_{e f f} \rho_{p} \frac{\partial W}{\partial r}=h_{m}\left(\left.\rho_{v}\right|_{r=r_{p}}-\rho_{\infty}\right)
$$

At $\mathrm{t}=0$

$$
W=0
$$

where $h_{m}$ is convective mass transfer coefficient and $\left.\rho_{v}\right|_{r=r_{p}}$ represents adsorptive density at the outer surface of the adsorbent particle which is in equilibrium with the adsorbate concentration. The following dimensionless parameters can be used to obtain the dimensionless form of the mass diffusion equation and related initial and boundary conditions;

$$
W^{*}=\frac{W-W_{i}}{W_{\infty}-W_{i}} ; r^{*}=\frac{r}{r_{p}} ; \tau=\frac{D_{e f f} t}{r_{p}^{2}} ; \rho_{v}^{*}=\frac{\rho_{v}}{\rho_{\infty}}
$$

By using the above dimensionless parameters, the dimensionless form of mass diffusion equation for the considered spherical adsorbent particle becomes as:

$$
\frac{\partial W^{*}}{\partial \tau}=\frac{1}{r^{* 2}} \frac{\partial}{\partial r^{*}}\left(r^{* 2} \frac{\partial W^{*}}{\partial r^{*}}\right)
$$

The dimensionless initial and boundary conditions for the problem can be written as:

At

$$
\frac{\partial W^{*}}{\partial r^{*}}=0
$$$$
r^{*}=0
$$

At $r^{*}=1$

$$
\frac{\partial W^{*}}{\partial r^{*}}=B i_{m}^{\prime}\left(1-\left.\rho_{v}^{*}\right|_{r^{*}=1}\right)
$$

At

$$
W^{*}=0
$$

The left side of Eq. (10) depends $W^{*}$ while the right side contains $\rho_{v}^{*}$. Hence, an additional relation is necessary to find $\rho_{v}^{*}$ in terms of $W^{*}$. The dimensionless isotherm equation (Eq. (1)) can be used to establish the required relation.

$B i_{m}$ is mass transfer Biot number. It is different than classical mass transfer Biot number and it can be calculated from the following equation;

$B i_{m}=B i_{m} G$

where, $B i_{m}$ is the classical mass transfer Biot number. $B i_{m}$ and $\mathrm{G}$ are defined as;

$B i_{m}=\frac{h_{m} r_{p}}{D_{e f f}}$

$G=\frac{\rho_{\infty}}{\rho_{p}\left(W_{\infty}-W_{i}\right)}$ 
In order to specify the range of $B i_{m}$, theoretical calculations are performed. For instance, the values of $B i_{m}$ for a commercial silica gel - water pair at $\mathrm{T}=303 \mathrm{~K}$ is found as 254.1 when $r_{p}=1.25 \mathrm{~mm}$ and $0.01 \mathrm{~m} / \mathrm{s}$. The performed theoretical studies showed that the considered range (from 0.5 to 750 ) of $B i_{m}$ can be faced in reality.

\section{SOLUTION METHOD}

The mass diffusion equation with initial and boundary conditions mentioned in the pervious section were solved numerically by finite difference method. The governing equations are linear however the condition for outer boundary condition is nonlinear (Eq. (10)). An inner iteration was performed to obtain the value of $W^{*}$ at the outer surface of the adsorbent particle by using the dimensionless isotherm. The convergence criterion for inner iteration was;

$\left|W^{*}\right|_{r^{*}=1}^{k+1}-\left.W^{*}\right|_{r^{*}=1} ^{k} \mid<10^{-7}$

where, $k$ is step number. It was assumed that the adsorption process was completed when $\bar{W}^{*}=0.99$. The duration which was required for adsorbate concentration to be attained from $\bar{W}^{*}=0$ to $\bar{W}^{*}=0.99$ was called as total dimensionless period of adsorption. The average adsorbate concentration (i.e., $\bar{W}^{*}$ ) was calculated numerically. Number of nodes in radial direction was 200.

\section{RESULTS AND DISCUSSION}

The results of the dimensionless mass diffusion equation (Eq. (8)) under initial and boundary conditions (Eqs. (9-11)) are obtained and presented in this section. It should be mentioned that the following results and discussions are valid for an isothermal gas adsorption in an adsorbent particle when Fick's law is valid, diffusion from surface to the center of particle occurs only by solid or surface diffusion and finally when the mass transfer diffusivity is constant.

\subsection{Intraparticle Diffusion Controlling}

The intraparticle diffusion controlling refers to the negligible effect of convective external mass transfer resistance on transfer rate. This kind of mass transfer mechanism occurs in the particles with large values of mass transfer Biot number. For the large values of mass transfer Biot number, a negligible gradient of adsorptive density exists at the outer region of adsorbent particle (i.e., $\left.W^{*}\right|_{r^{*}=1} \approx 1$ ). Therefore, the boundary condition at the outer surface of the particle become as:

at $r^{*}=1$;

$W^{*}=1$

For a case with intraparticle diffusion controlling, the solution of Eq. (8) does not depend on any dimensionless parameter, and the dimensionless uptake curve for all shapes of isotherms is expected to be identical. The solution of Eq.
(8) was obtained and dimensionless uptake curve is shown in Fig. (6). Since Eq. (8) and its initial and boundary conditions for a case with intraparticle diffusion controlling are linear, its analytical solution is also possible. Fig. (6) shows the comparison of the analytical solution of Eq. (8) [7] with the obtained numerical results of this study. A good agreement between two results can be observed. Fig. (6) also presents the accuracy of the present numerical study. It shows that the dimensionless adsorbate concentration attains to 1 after a dimensionless period of 0.42 (i.e., $\tau_{\text {total }}=0.42$ ). This total dimensionless adsorption period is valid for all isotherm shapes when intraparticle mass transfer resistance controls adsorption process.

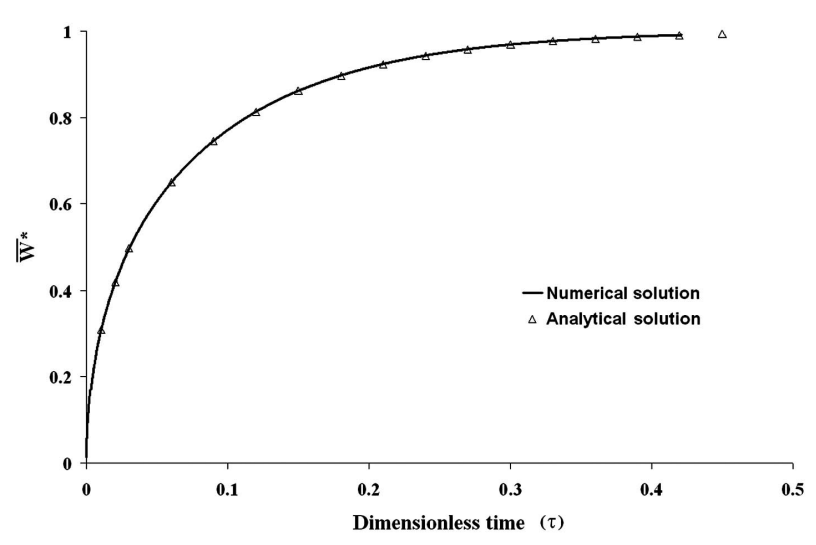

Fig. (6). Dimensionless uptake for an adsorbent particle with intraparticle diffusion controlling.

\subsection{Effect of External Mass Transfer Resistance}

The solution of the mass transfer equation (Eq. (8)) with the initial and boundary conditions (Eq. (9-11)) were obtained for different values of $B i_{m}^{\prime}$ and various shapes of isotherms. The uptake curves for different values of $B i_{m}^{\prime}$ $\left(B i_{m}^{\prime}=100,20,1\right.$ and 0.5$)$ when $\mathrm{n}=0.05$ are illustrated in Fig. (7). For $n=0.05$, the isotherm has convex shape and dimensionless adsorbate concentration can attain to maximum equilibrium value (i.e. $W^{*}=1$ ) at a low dimensionless density. As seen from the Fig. (7), the variation of the average adsorbate concentration with time for $B i_{m}^{\prime}=20$ and 100 are identical. For these values of $B i_{m}^{\prime}$, the external mass transfer resistance is not the controlling parameter. However, Fig. (7) shows that the adsorption period is highly influenced from $B i_{m}^{\prime}$ for the cases such as $B i_{m}^{\prime}=1$ and 0.5 . The decrease in $B i_{m}^{\prime}$ refers to the increase of external mass transfer resistance, and that is why the total adsorption period increases for the cases with $B i_{m}^{\prime}=1$ and 0.5 . The total adsorption period for an adsorbent particle with $B i_{m}^{\prime}=20$ is $\tau=0.42$, however the decrease of $B i_{m}^{\prime}$ from 20 to 0.5 prolongs the total adsorption period to $\tau=0.92$. 


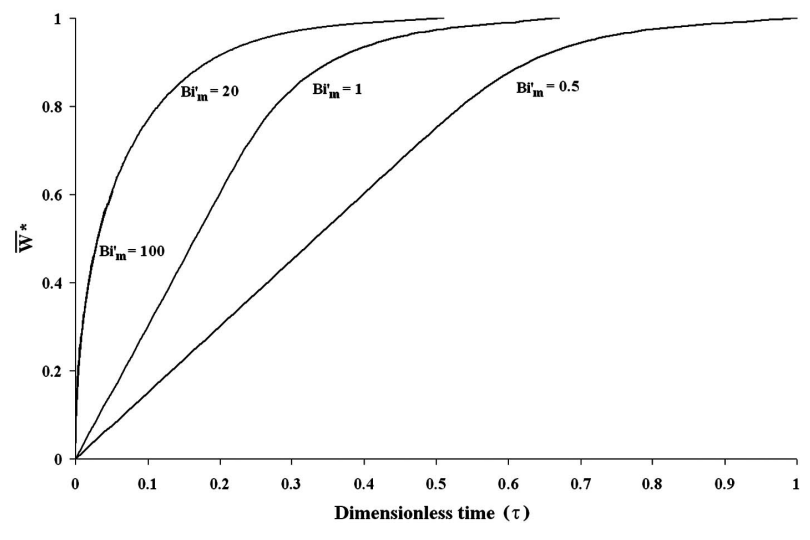

Fig. (7). The changes in average adsorbate concentration for different values of $B i_{m}^{\prime}$ when $\mathrm{n}=0.05$.

In order to determine a critical mass transfer Biot number (i.e., $B i_{m, c r}^{\prime}$ ), Eq. (8) was solved for different values of $B i_{m}^{\prime}$ and the obtained results were compared with the results of case without external mass transfer resistance shown in Fig. (6). It was observed that the changes in average adsorbate concentration are almost identical at $90 \%$ of adsorption process period when $B i_{m, c r}^{\prime}>15$ for an isotherm with $\mathrm{n}=$ 0.05 . The following criterion was used to determine $B i_{m, c r}$ (for $90 \%$ adsorption period):

$$
\left|\bar{W}^{*}\left(\tau, n, B i_{m}^{\prime}\right)-\bar{W}^{*}(\tau, n)\right|<0.05
$$

where $\bar{W}^{*}\left(\tau, n, B i_{m}^{\prime}\right)$ is the average adsorbate concentrations of the particle with mass transfer Biot number of $B i_{m}^{\prime}$ and an isotherm shape represented by $\mathrm{n} . W^{*}(\tau, n)$ is the average adsorbate concentration for a case with negligible external mass transfer resistance.

The changes in local adsorbate concentration with time for $B i_{m}^{\prime}=20$ and 0.5 are shown in Fig. (8a) and (b), respectively when $\mathrm{n}=0.05$. The local adsorbate concentration in the particle is zero at the beginning of adsorption process. As seen from Fig. (8a), after a short period of $\tau=0.01$, the adsorbate concentration at the surface of adsorbent particle reaches the final equilibrium concentration (i.e., $W^{*}=1$ ). The adsorbate is diffused to the center of particle and the local adsorbate concentration increases over time. After approximately $\tau=0.4$, the adsorbate concentration in the entire particle attains the equilibrium state. Fig. (8b) shows the local adsorbate concentration in the particle with $B i_{m}^{\prime}=0.5$ for the same value of $(n=0.05)$. The local adsorbate concentration in the particle is initially zero. After $\tau=0.01$, the adsorbate concentration at the outer surface of adsorbent particle becomes 0.063 (i.e., $W^{*}=0.063$ ) which is less than $W^{*}=1$ due to the existence of external mass transfer resistance. The adsorbate concentration increases over time not only at the surface but also at center region of adsorbent particle. After $\tau=0.92$, the average adsorbate concentration of the adsorbent particle approximately attains the final value which is $W^{*}=1$.

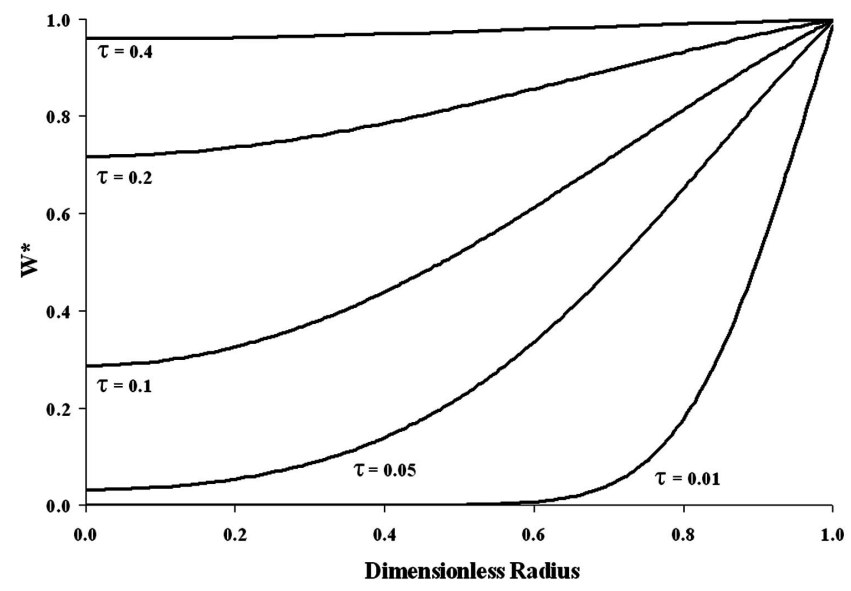

(a)

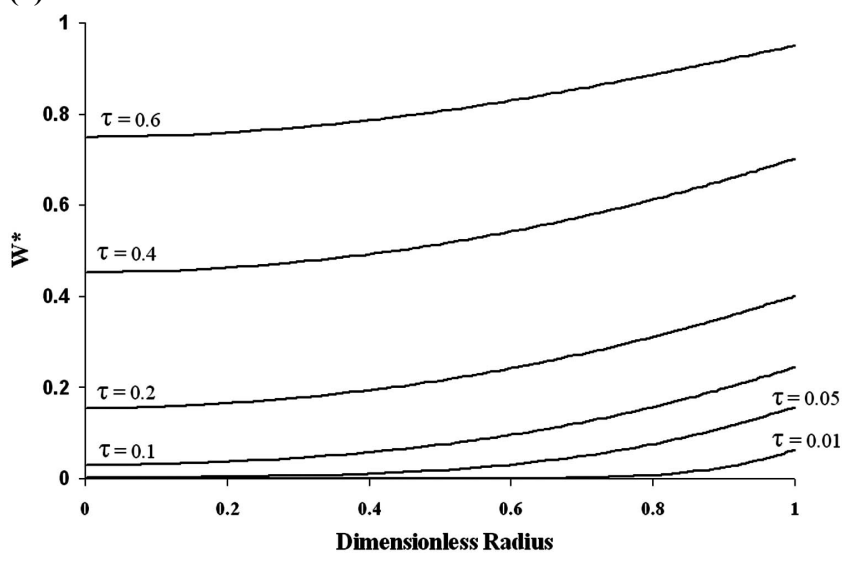

(b)

Fig. (8). The change in local adsorbate concentration with time a) $\left.B i_{m}=20, \mathrm{n}=0.05 ; \mathrm{b}\right) B i_{m}=0.5, \mathrm{n}=0.05$.

The changes in average adsorbate concentration over time for adsorbent-adsorbate pairs with isotherm of $n=20$ and $B i_{m}^{\prime}=200,100,20,1,0.5$ are shown in Fig. (9). The uptake curves of $B i_{m}^{\prime}=100$ and 200 are close to each other. The obtained results show that the average adsorbate concentrations are almost identical at least for $90 \%$ of adsorption period for the cases with mass transfer Biot number greater than 750 (i.e., $B i_{m, c r}^{\prime}=750$ ). The decrease of $B i_{m}^{\prime}$ extends the period of adsorption due to increase in the external mass transfer resistance. For $n=20$, the dimensionless adsorption period for an adsorbent particle with $B i_{m}^{\prime}=100$ is $\tau=0.66$, and the decrease of $B i_{m}^{\prime}$ from 100 to 0.5 prolongs the period of adsorption to $\tau=53.71$.

The effects of isotherm shape on the changes of average adsorbate concentration with dimensionless time can be seen from Fig. (10) when $B i_{m}^{\prime}=1$. As seen, the average adsorbate concentration for isotherm with $\mathrm{n}=0.05$ (convex shape) reaches final equilibrium value faster than the other iso- 


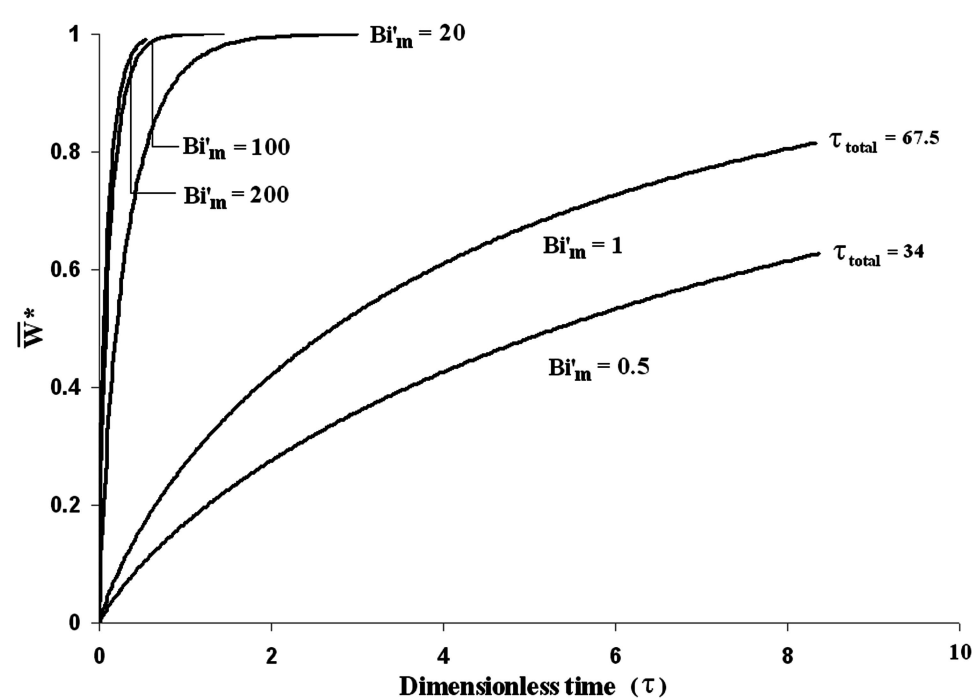

Fig. (9). The changes in average adsorbate concentration with time when $\mathrm{n}=20$ and $B i_{m}^{\prime}=200,100,20,1,0.5$.

therms. By increasing value of $\mathrm{n}$ from 0.05 to 20 (i.e., change of isotherm shape from convex to concave), the dimensionless time required for the adsorbate concentration to reach the equilibrium is highly prolonged.

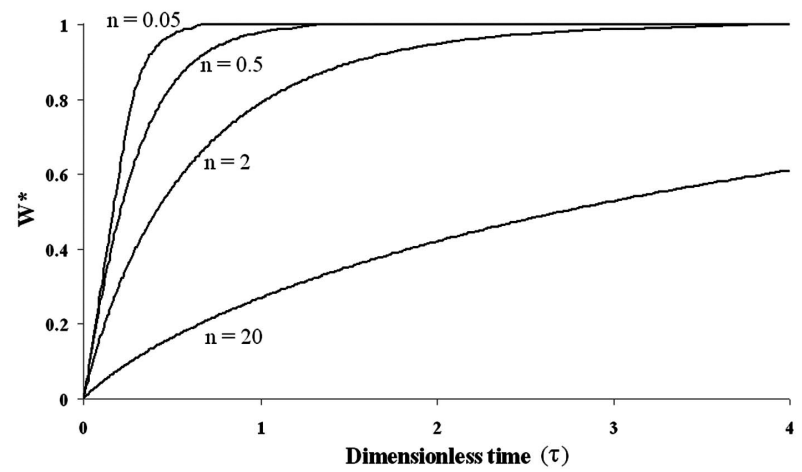

Fig. (10). The change in average dimensionless concentration with dimensionless time for different shapes of isotherm when $B i_{m}^{\prime}=1$.

Fig. (11) shows the variation of total dimensionless adsorption period with the dimensionless shape of isotherm for different values of $B i_{m}^{\prime}$. As seen for $B i_{m}^{\prime}=750$, the adsorption period is short (around $\tau=0.42$ ) and it is not influenced by the shape of isotherm. By the decrease in $B i_{m}^{\prime}$ from 750 to 20 , the total adsorption period is not changed for isotherm with $\mathrm{n}=0.05$. However, an increase in adsorption period for dimensionless isotherm with $\mathrm{n}=20$ can be observed. Further increase in effect of external mass transfer resistance extends the period of adsorption and the total dimensionless period is considerably affected from the shape of isotherm when $B i_{m}^{\prime}=0.5$. For the range of $B i_{m}^{\prime}$ and shapes of isotherms studied in the present work, the maximum total dimensionless adsorption period was observed for an adsorbent particle with $\mathrm{n}=20$ and $B i_{m}^{\prime}=0.5$ (i.e., $\tau_{\text {total }}=$ 53.71).

Based on criterion defined by Eq. (17), the critical mass transfer Biot number was determined for different isotherm shapes and the results are presented in Fig. (12). As seen, the value of critical Biot number depends on isotherm shape. The value of critical Biot number increases by change of isotherm shape from convex to concave shape. For an

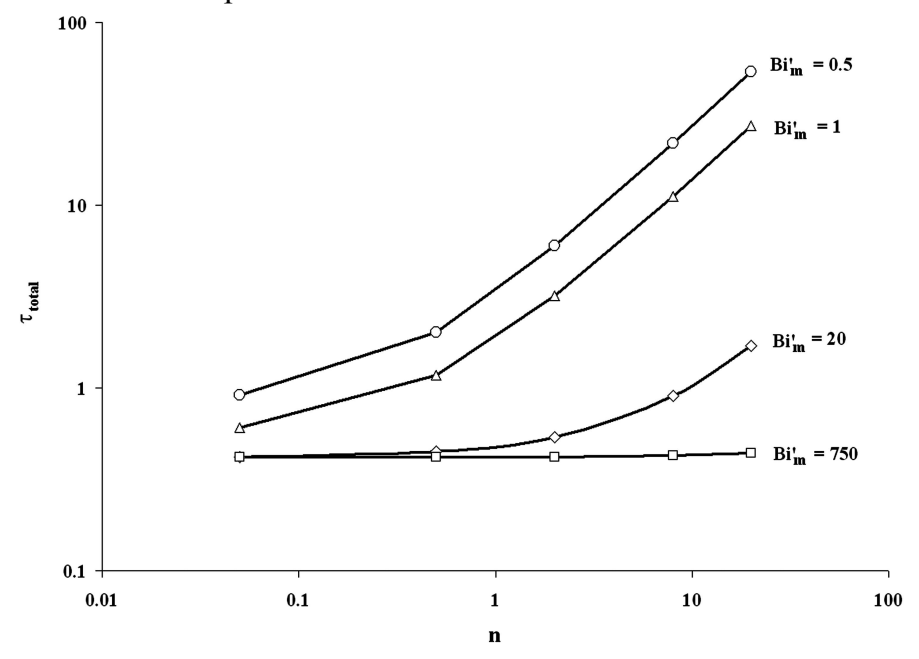

Fig. (11). The change in total dimensionless adsorption period with shape of the isotherm for different values of $B i_{m}^{\prime}$. 
isotherm with $\mathrm{n}=0.05$, the value of critical mass transfer Biot number is $B i_{m}^{\prime}=15$, and this value increases to $B i_{m}^{\prime}=$ 750 for $n=20$.

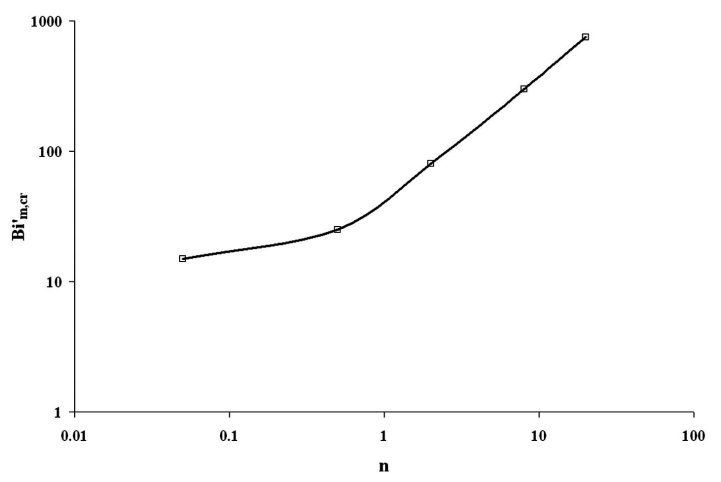

Fig. (12). Critical mass Biot number for different isotherm shapes.

\section{CONCLUSIONS}

The dimensionless form of mass diffusion equation for a spherical adsorbent particle located in an infinite adsorptive medium was solved for different shapes of isotherms and different values of mass transfer Biot number. The effective diffusivity was assumed constant and Fick's law is valid to simulate surface diffusion due to adsorbate concentration gradient in the particle. Based on the obtained results, it was found that the total dimensionless adsorption period is not influenced from the shape of isotherm for the cases with mass transfer Biot number greater than critical mass Biot number. However, the total dimensionless adsorption period is considerably affected from the shape of isotherm for the cases with mass transfer Biot number less than $B i_{m, c r}^{\prime}$. When $B i_{m}^{\prime}<B i_{m, c r}^{\prime}$, the dimensionless adsorption period is considerably shorter for convex isotherms ( such as $\mathrm{n}=0.05$ ) compared to concave one (such as $n=20$ ). For a mass Biot number of 20 , the total dimensionless period of adsorption for an isotherm with convex shape (i.e., $\mathrm{n}=0.05$ ) is $\tau=0.42$, this value extends to $\tau=1.71$ for an adsorbentadsorbate pair with concave isotherm shape (i.e., $\mathrm{n}=20$ ). The critical mass Biot numbers for different shapes of isotherms were also calculated. The critical mass Biot number (i.e., $B i_{m, c r}^{\prime}$ ) for an isotherm with $\mathrm{n}=0.05$ was found as 15 and the value of $B i_{m, c r}^{\prime}$ increases to 750 for an isotherm with $\mathrm{n}=20$.

The results of present study showed that the shape of isotherm should be taken into account for the cases in which the external mass transfer plays an important role on mass transport. For those cases, the adsorbent-adsorbate pairs with convex shape isotherm should be selected in order to have shorter adsorption period. The present study can be extended and performed for macropore controlled intraparticle diffusion and non-isothermal adsorption processes.

\section{NOMENCLATURE}

$B i_{m}=$ Mass transfer Biot number (Eq. (12))

$D=\operatorname{Diffusivity}\left(\mathrm{m}^{2} / \mathrm{s}\right)$

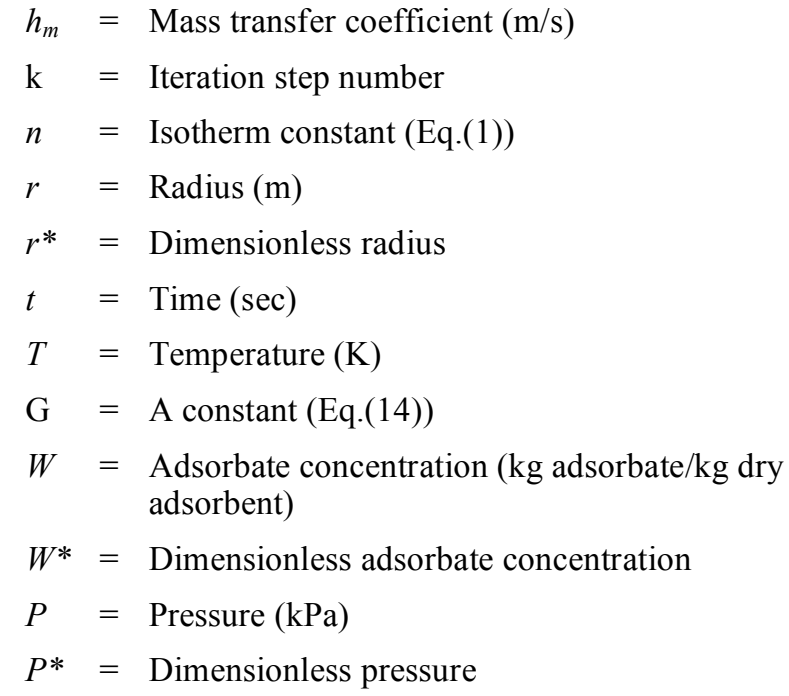

Greek Symbols

$\rho=$ Density $\left(\mathrm{kg} / \mathrm{m}^{3}\right)$

$\rho^{*}=$ Dimensionless density

$\tau=$ Dimensionless time

\section{Subscripts}

$\begin{aligned} s & =\text { Surface } \\ p & =\text { Particle } \\ \mathrm{v} & =\text { Vapor, gas } \\ \infty & =\text { Equilibrium state } \\ \text { eff } & =\text { Effective } \\ i & =\text { Initial } \\ \text { sat } & =\text { Saturation }\end{aligned}$

\section{ACKNOWLEDGEMENT}

None declared.

\section{CONFLICT OF INTEREST}

None declared.

\section{REFERENCES}

[1] Y. Aristov, "Novel materials for adsorptive heat pumping and storage: screening and nanotailoring of sorption properties", $J$. Chem. Eng. Jpn., vol. 40, pp. 1242-1251, 2007.

[2] S. Ülkü, and M. Mobedi, Ed. Adsorption in Energy Storage. Kluwer Academic Publishers, The Netherland 1989.

[3] Z. E. Yildirim, M. Mobedi, and S. Ülkü, "A Review on Proper Working Pairs for Solar Adsorption Heat Pumps", in Solar Future Conference, Istambul, Turkey 2010, pp. 195-202.

[4] C. T. Erkey, C. Erkey, and A. Akgerman, "Mathematical modeling of adsorption and desorption of volatile contaminants from soil: influence of isotherm shape on adsorption and desorption profiles", Environ. Sci. Technol., vol. 30, pp. 2127-2134, 1996.

[5] J. Y. San, and W. M. Lin, "Comparison among three adsorption pairs for using as the working substances in a multi-bed adsorption heat pump", Appl. Therm. Eng., vol. 28, pp. 988-997, 2008.

[6] C. Nobrega, and N. Brum, "Influence of isotherm shape over desiccant cooling cycle performance", Heat Transf. Eng., vol. 30, pp. 302-308, 2009. 
[7] J. Karger, and D. M. Ruthven, Diffusion in Zeolites and other Microporous Solids, New York: Wiley-Interscience, 1992.

[8] D. M. Ruthven, Principles of Adsorption and Adsorption Processes, USA: Wiley-Interscience, 1984.

[9] M. Suzuki, Adsorption Engineering, Tokyo: Elsevier, 1990.

[10] F. Rouquerol, F. Rouquerol, and K. S. W. Sing, Adsorption by Powders and Porous Solids, London: Academic Press, 1999.

[11] S. J. Gregg, and K. S. W. Sing, Adsorption, Surface Area and Porosity, London: Academic Press, 1982.
[12] K. Daou, R. Wang, G. Yang, and Z. Xia, "Theoretical comparison of the refrigerating performances of a $\mathrm{CaCl}_{2}$ impregnated composite adsorbent to those of the host silica gel", Int. J. Thermal Sci., vol. 47-1, pp. 68-75, 2008.

[13] Z. Tamainot-Telto, S. J. Metcalf, R. E. Critoph, Y. Zhong, and R. Thorpe "Carbon-ammonia pairs for adsorption refrigeration applications: ice making, air conditioning and heat pumping", Int. J. Refrig., vol. 32-6, pp. 1212-1229, 2009.

Received: May 30, 2011

Revised: August 03, 2011

Accepted: August 04, 2011

(C) Yildirim et al.; Licensee Bentham Open.

This is an open access article licensed under the terms of the Creative Commons Attribution Non-Commercial License (http://creativecommons.org/ licenses/by-nc/3.0/), which permits unrestricted, non-commercial use, distribution and reproduction in any medium, provided the work is properly cited. 